\title{
Generic Coexistence of Fermi Arcs and Dirac Cones on the Surface of Time-Reversal Invariant Weyl Semimetals
}

\author{
Alexander Lau, ${ }^{1}$ Klaus Koepernik, ${ }^{1}$ Jeroen van den Brink, ${ }^{1,2}$ and Carmine Ortix ${ }^{3,1}$ \\ ${ }^{1}$ Institute for Theoretical Solid State Physics, IFW Dresden, 01171 Dresden, Germany \\ ${ }^{2}$ Institute for Theoretical Physics, TU Dresden, 01069 Dresden, Germany \\ ${ }^{3}$ Institute for Theoretical Physics, Center for Extreme Matter and Emergent Phenomena, \\ Utrecht University, Princetonplein 5, 3584 CC Utrecht, Netherlands
}

(Received 6 January 2017; revised manuscript received 13 July 2017; published 18 August 2017)

\begin{abstract}
The hallmark of Weyl semimetals is the existence of open constant-energy contours on their surface-the so-called Fermi arcs — connecting Weyl points. Here, we show that, for time-reversal symmetric realizations of Weyl semimetals, these Fermi arcs, in many cases, coexist with closed Fermi pockets originating from surface Dirac cones pinned to time-reversal invariant momenta. The existence of Fermi pockets is required for certain Fermi-arc connectivities due to additional restrictions imposed by the six $\mathbb{Z}_{2}$ topological invariants characterizing a generic time-reversal invariant Weyl semimetal. We show that a change of the Fermi-arc connectivity generally leads to a different topology of the surface Fermi surface and identify the half-Heusler compound LaPtBi under in-plane compressive strain as a material that realizes this surface Lifshitz transition. We also discuss universal features of this coexistence in quasiparticle interference spectra.
\end{abstract}

DOI: 10.1103/PhysRevLett.119.076801

Introduction.-Sparked by the discovery of the quantum Hall effect and its theoretical explanation [1-3], the study of topological phases of matter has been one of the driving forces in modern condensed matter physics [4-9]. Novel states of matter that emerged from these investigations are, for instance, time-reversal invariant (TRI) topological insulators [10-15], and crystalline topological insulators [16-19], which are realized in several material systems. In recent years, the family of topological materials has been extended by topological semimetals [7]. A milestone was the experimental discovery of Weyl semimetals (WSMs) [20-23]. WSMs are three-dimensional gapless materials whose bulk energy bands cross linearly at isolated points, the so-called Weyl nodes, in the Brillouin zone (BZ) [24-28]. Weyl nodes are characterized by their chirality and can only be annihilated pairwise. For this reason, they are a robust bulk feature [25,29]: generic perturbations shift the nodes in energy and momentum space without annihilating them. Most importantly, WSMs host robust surface states commonly referred to as Fermi arcs. They form an open Fermi surface connecting the surface projections of Weyl nodes with opposite chiralities [24].

Recently, it has been theoretically proposed that Fermi arcs can, under certain conditions, coexist with surface Dirac cones at the interface between a time-reversal broken WSM and a three-dimensional (3D) TRI topological insulator [30,31]. The close relation between Dirac cones and Fermi arcs is also manifest in the fact that Dirac cones can be created by fusing Weyl points in a specific manner [29]. In this Letter, we show that in time-reversal symmetric WSMs, the coexistence of Dirac cones and Fermi arcs arises naturally. It is due to additional restrictions on the surface Fermi surface imposed by the $\mathbb{Z}_{2}$ invariants associated with the TRI planes of the WSM. We, thereby, show that this coexistence is encountered in the half-Heusler compound LaPtBi, which realizes a Weyl semimetal phase under in-plane compressive strain [32]. Finally, we determine universal features of this coexistence in quasiparticle-interference (QPI) spectra, which are relevant for scanning tunneling experiments [33-37].

$\mathbb{Z}_{2}$ invariants in TRI Weyl semimetals. - It is well known that the existence of Weyl nodes in momentum space requires either time-reversal $\Theta$ or inversion $\mathcal{I}$ symmetry breaking $[25,26,29]$. In the presence of both symmetries, all energy bands are at least doubly degenerate, which requires a linear crossing to be a fourfold degenerate Dirac point. $\Theta$ or $\mathcal{I}$ symmetry-breaking perturbations split a Dirac point into two separate Weyl points of opposite chirality. A Weyl node represents a monopole of the Berry flux $\mathcal{A}(\mathbf{k})$ in momentum space [24,29]. Consequently, an integral of the Berry flux over a closed surface enclosing the Weyl node results in a nonzero integer value, which defines the topological charge or chirality of the Weyl node [24,29]. Since the total topological charge in the whole BZ must vanish [38], Weyl nodes always appear in pairs of opposite chirality. Moreover, in TRI systems, each Weyl point has a time-reversal partner of the same chirality, which implies the total number of Weyl nodes in these systems to be $4 n$, with $n$ being an integer [29]. The nonzero topological charge of the Weyl nodes can also be interpreted [25] as the change in the Chern number of the collection of gapped two-dimensional (2D) systems realized by decomposing the 3D BZ of a WSM in 2D momentum space cuts separating the Weyl points from each other [see Fig. 1(a)]. This property is at the basis of the existence of one of the most interesting hallmarks of WSMs: the existence of open 
constant-energy contours in the surface BZ called Fermi $\operatorname{arcs}[24]$.

In general, time-reversal invariant WSMs can be additionally characterized by six $\mathbb{Z}_{2}$ invariants [39], except when the Weyl points are pinned to time-reversal invariant momenta, which can only occur in materials with chiral space groups [40]. The Chern number of the effective 2D insulators realized by the TRI planes will be zero, but the time-reversal polarizations still allow one to characterize the effective $2 \mathrm{D}$ systems in terms of a $\mathbb{Z}_{2}$ topological invariant $\nu$ [41]. Contrary to TRI insulators in $3 \mathrm{D}$, where the six $\nu_{i}$ are not independent and can be reduced to four using homotopy arguments [42,43]-the well-known strong and weak indices of 3D TRI insulators [11,41] in a time-reversal invariant WSM, all six $\mathbb{Z}_{2}$ invariants are independent and characterize the WSM as explained below.

For a generic surface of a WSM, by bulk-boundary correspondence, the $\nu_{i}$ determine whether an even $\left(\nu_{i}=0\right)$ or odd $\left(\nu_{i}=1\right)$ number of Kramers pairs of surface states cross the Fermi level along the surface projection of the $i$ th TRI plane. This imposes restrictions on the structure of the surface Fermi surface but still does not uniquely determine it. Figures 1(c) and 1(d) sketch two allowed, but qualitatively very different, surface Fermi surfaces of a timereversal invariant WSM in which the $\mathbb{Z}_{2}$ invariants of the

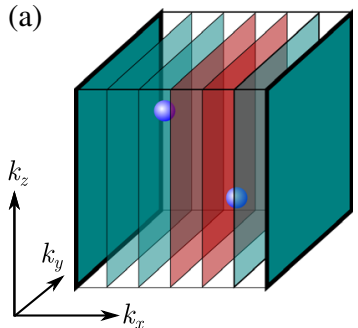

(c)

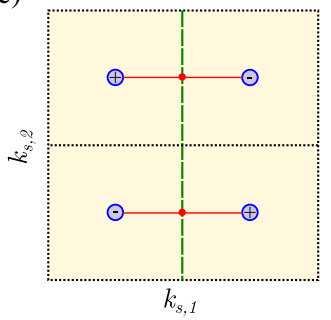

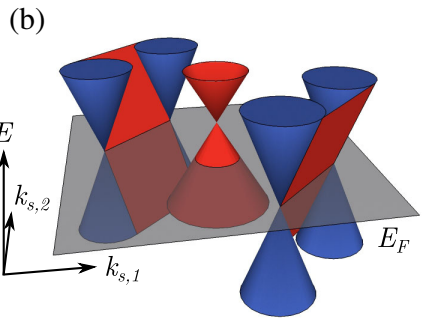

(d)

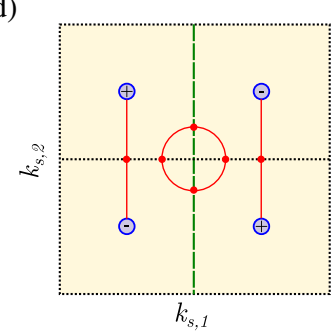

FIG. 1. (a) The BZ of a WSM as a collection of 2D insulators with zero (green) or nonzero (red) Chern numbers. Weyl nodes (blue spheres) separate planes with different Chern numbers. The bold frames indicate the TRI 2D insulators characterized by a $\mathbb{Z}_{2}$ invariant. (b) Typical low-energy surface spectrum of a TRI WSM with an additional surface Dirac cone: surface states are shown in red, whereas the surface projections of the 3D bulk Weyl cones are highlighted in blue. (c)-(d) Fermi arc connectivities in the surface BZ of a TRI WSM with four Weyl points indicated by their topological charge \pm . The surface projections of the 3D TRI planes are highlighted by dotted black $(\nu=0)$ or dashed green $(\nu=1)$ lines. planes $k_{s, 2}=0, \pi$ and $k_{s, 1}=\pi$ have the trivial value 0 , whereas the invariant associated with the plane at $k_{s, 1}=0$ has the nontrivial value 1. A surface Fermi surface consisting of only two open arcs, connecting Weyl points as depicted in Fig. 1(c), is entirely allowed. However, different pairs of Weyl points of opposite chirality can be connected only if an additional Fermi pocket, enclosing a time-reversal invariant point, is created [see Fig. 1(d)]. The latter situation is a unique signature of Fermi arcs coexisting with a surface Dirac cone [see Fig. 1(b) and Ref. [44] ], which is an exclusive feature of TRI Weyl semimetals. This surface Dirac cone is protected for a given connectivity of the Fermi arcs since its corresponding Fermi pocket can only be removed by connecting it to the Fermi arcs, which would lead to another reconnection of the Weyl nodes. We emphasize that, while this transition does not change the $\mathbb{Z}_{2}$ invariants of the time-reversal invariant WSM, the change of the Fermi surface topology does imply a Lifshitz transition on the surface of the material $[45,46]$. Before studying this Lifshitz transition in an explicit Hamiltonian, first, we determine the generic consequences for QPI. We compare this to explicit QPI calculations for the explicit Hamiltonian later on.

Phenomenological QPI patterns.-Having established the coexistence of Fermi arcs and Dirac cones in timereversal invariant WSMs, we now proceed to analyze their fingerprints in QPI patterns, which can be observed in scanning tunneling spectroscopy experiments [33-37]. We start out by noticing that QPI spectra can be approximated in terms of the joint density of states (JDOS) [33,47]

$$
J(\mathbf{q}, E)=\int d^{2} k A(\mathbf{k}+\mathbf{q}, E) A(\mathbf{k}, E),
$$

where $\mathbf{k}$ is the momentum parallel to the surface, and $A(\mathbf{k}, E)=-1 / 2 \pi \operatorname{Im}\left\{\operatorname{Tr}\left[G_{s}(\mathbf{k}, E)\right]\right\}$ is the spectral function with the surface Green's function $G_{s}(\mathbf{k}, E)$.

To understand the characteristic features arising in the JDOS, we have performed a phenomenological analysis based on a simple ansatz for the spectral function. We present the details of our calculation in the Supplemental Material [44] and proceed here with the presentation of the key results. First of all, our analysis reproduces the results of previous studies, namely a pinch point at $\mathbf{q} \equiv 0$ [34] surrounded by crescent-shaped patterns due to the contribution of the Fermi arcs [48], and a disk feature associated with the presence of a Dirac cone [49]. Most importantly, for the case of coexistence, we find two additional kidneyshaped features at a distance $|\mathbf{q}|$ corresponding to the distance between the Fermi arcs and the Fermi pocket. Furthermore, the broadening of these patterns corresponds to the diameter of the Fermi pocket. We can unambiguously attribute this feature to scattering events between the Fermi pocket and Fermi arcs. Hence, they represent the universal 
QPI feature of the coexistence of Fermi arcs with a Dirac cone on the surface of time-reversal invariant WSMs.

Tight-binding model formulation.-Next, we introduce a tight-binding model for a time-reversal invariant WSM to investigate, on a microscopic basis, the coexistence of surface Dirac cones and Fermi arcs. The tight-binding model is defined on a cubic lattice and reads

$$
\begin{aligned}
H(\mathbf{k})= & a\left(\sin k_{x} \tau^{1} s^{3}+\sin k_{y} \tau^{2} s^{0}\right)+\beta \tau^{2} s^{2}+d \tau^{2} s^{3} \\
& +\left[t \cos k_{z}+2 b\left(2-\cos k_{x}-\cos k_{y}\right)\right] \tau^{3} s^{0} \\
& +\alpha \sin k_{y} \tau^{1} s^{2}+\lambda \sin k_{z} \tau^{0} s^{1},
\end{aligned}
$$

where the $s^{i}$ are Pauli matrices in spin space, whereas the $\tau^{i}$ are Pauli matrices associated with additional orbital degrees of freedom. The lattice constant has been set to unity. The Hamiltonian is based on a general tight-binding model introduced in Ref. [34]. The model preserves time-reversal symmetry with $\Theta=i \tau^{0} s^{2} K, \mathbf{k} \rightarrow-\mathbf{k}$, where $K$ is a complex conjugation. The $\beta, d$, and $\lambda$ terms break inversion symmetry with the inversion operator $P=\tau^{3} s^{0}, \mathbf{k} \rightarrow-\mathbf{k}$, which is a necessary condition for the existence of a Weyl semimetal phase.

To demonstrate the coexistence of surface Dirac cones and Fermi arcs in $H(\mathbf{k})$, we start from a particular Weyl semimetal phase and vary the parameter $\beta$. The results are presented in Fig. 2. With the chosen parameters, the model features four bulk Weyl points with a topological charge of \pm 1 , as can be determined by integrating the Berry flux of the bulk Hamiltonian in Eq. (2) over the surface of a BZ volume containing the Weyl node. The fact that the Weyl nodes are all located away from the TRI planes of the bulk BZ also allows one to calculate all six of the $\mathbb{Z}_{2}$ invariants using their Wannier-center formulation (see Refs. [44,50]). In particular, we find that $\nu_{k_{2}=\pi}=1$ while the remaining five $\mathbb{Z}_{2}$ invariants are zero. Therefore, at the (010) surface, we expect an odd number of Kramers pairs at $k_{z}=\pi$ and an even number of Kramers pairs at $k_{z}=0$ and $k_{x}=0, \pi$. For large values of $\beta$, we find that Fermi arcs connect two Weyl nodes in the left half-plane and two Weyl nodes in the right halfplane [see Fig. 2(a)]. The fact that the Fermi arcs cross only the line $k_{z}=\pi$ is in agreement with the values of the topological invariants. In Fig. 2(b), we show the corresponding JDOS based on the (010) surface Green's function of the system (see Refs. [44,51] for computational details). We see a pinch point surrounded by two crescent-shaped patterns in agreement with our general consideration.

By decreasing the parameter $\beta$, the Fermi arcs are bent towards each other until they intersect at a critical value of $\beta$ [see Fig. 2(c)]. This point corresponds to the Lifshitz transition at which the connectivity of the Fermi arcs changes. A further decrease in the parameter $\beta$ reveals the coexistence of Dirac cones and Fermi arcs [see Fig. 2(e)]: surface Fermi arcs connect two Weyl nodes in the upper half-plane and two Weyl nodes in the lower
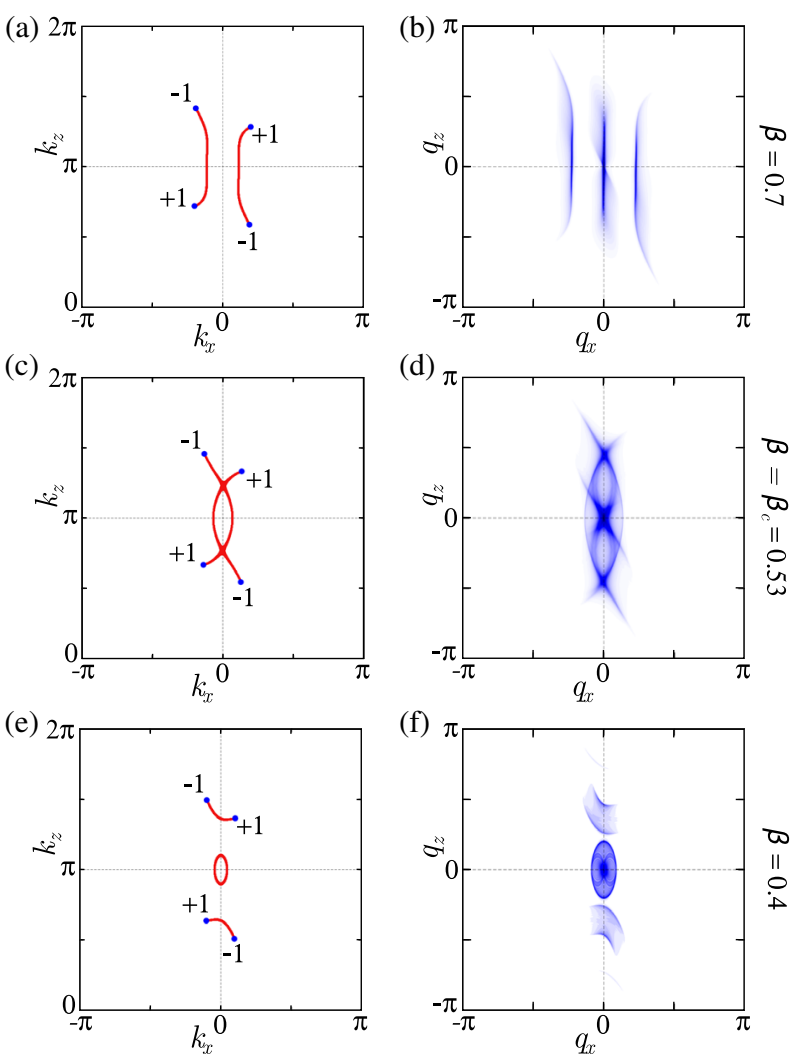

FIG. 2. Fermi surfaces and JDOS for (010) surfaces in the tightbinding model with $a=b=1, t=1.5, \alpha=0.3, d=0.1$, $\lambda=0.5$, and $E_{F}=0$ : (a),(c),(e) the first column shows the Fermi surfaces for different values of the parameter $\beta$. The bulk Weyl nodes are highlighted in blue and their topological charge is indicated. Surface states are highlighted in red. (b),(d),(f) The second column shows the corresponding JDOS spectra. In (f), the kidney-shaped features indicative of the coexistence of Fermi arcs and Dirac cones are clearly visible in the JDOS.

half-plane of the surface BZ. In addition to the open-arc features, we find an elliptical Fermi pocket of surface states around the $\bar{Z}$ point of the surface BZ. The existence of the Fermi pocket is required for this particular connectivity of Weyl nodes to satisfy the number of surface states imposed by the topological invariants $\nu_{i}$ which have not changed during the Lifshitz transition. From an analysis of the surface band structure [44], we find that the Fermi pocket originates from a dangling surface Dirac cone. This type of Dirac cone connects bulk conduction to bulk valence bands not along all directions of the surface BZ, as would be the case for a 3D topological insulator. This is only possible in TRI Weyl semimetals due to the lack of a global relation between the $\mathbb{Z}_{2}$ invariants.

The JDOS of this configuration is in perfect agreement with our analytical considerations (see Ref. [44]): we find a new elliptical feature around the origin associated with the additional Fermi pocket. The characteristic crescentshaped Fermi-arc features are barely visible due to the small size of the Fermi arcs. Most importantly, we find the 

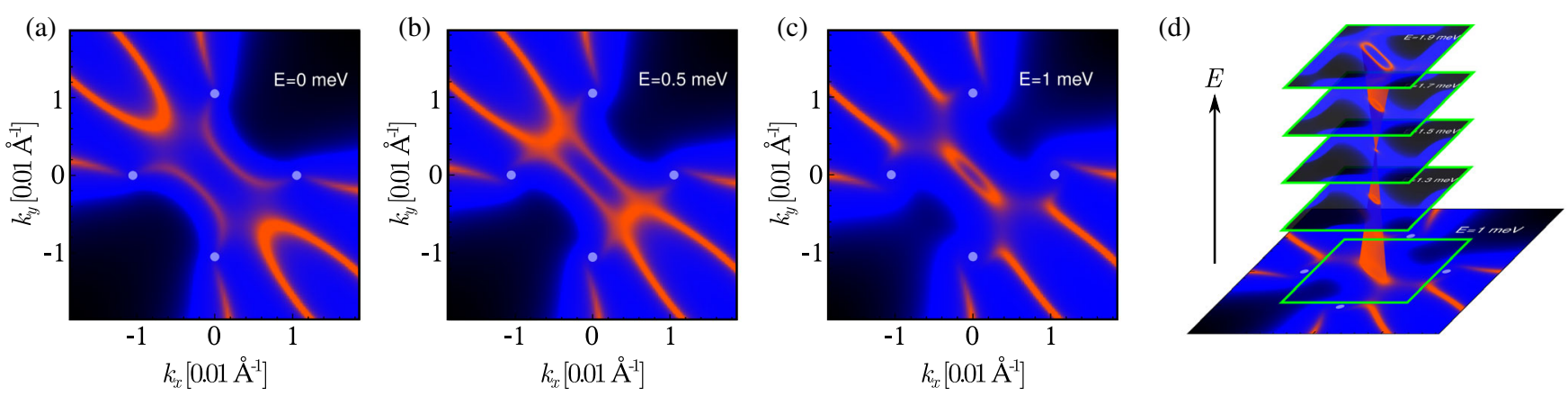

FIG. 3. Surface Fermi surfaces of LaPtBi with (001) termination: shown is the surface spectral weight. The positions of the four Weylpoint projections are marked by grey dots. The panels (a)-(c) display the transition between different Fermi-arc connectivities by varying the Fermi level. Note that the connectivity shown in (c) requires the presence of an additional Fermi pocket around the origin which resembles Fig. 2(e). (d) A further increase of the Fermi level reveals that the Fermi pocket, indeed, originates from a Dirac cone around $\bar{\Gamma}$.

kidney-shaped features indicative of scattering between the Fermi arcs and the Fermi pocket. Their broadening equals the size of the Fermi pocket whereas their position corresponds to the distance between Fermi pocket and Fermi arcs. A comparison of Figs. 2(b) and 2(f) shows that the different Fermi-arc connectivities are reflected in distinct universal JDOS features.

Strained LaPtBi.-Having established the coexistence of Dirac cones and Fermi arcs in a generic tight-binding model of TRI Weyl semimetals, we next show its realization in the half-Heusler compound $\mathrm{LaPtBi}$, which possesses both band inversion [52] and lattice noncentrosymmetry. A recent theoretical $a b$ initio study suggests that, under a broad range of in-plane biaxial compressive strain, LaPtBi realizes a Weyl semimetal phase with eight Weyl nodes residing precisely at the Fermi level at stoichiometry composition [32]. We have performed density functional theory calculations employing the full potential local orbital method [53] in four-component relativistic mode (see Ref. [44] for computational details), applying a compressive in-plane strain of $a=0.99 a_{0}, c=1.02 a_{0}$. For the study of surface states, we investigate a semiinfinite slab with a (001) surface corresponding to a termination along one of the $\mathrm{LaBi}$ planes.

Because of the concomitant presence of time-reversal and twofold rotations along the $x$ and $y$ axes, eight Weyl points of charge \pm 1 are located at the $k_{x}=0$ and $k_{y}=0$ planes of the bulk BZ. Moreover, the electronic states in the $k_{x}=k_{y}$ and $k_{x}=-k_{y}$ planes are all gapped, so that the $\mathbb{Z}_{2}$ topological invariant in these TRI subsystems is well defined. In the (001) surface BZ, the Weyl points are projected pairwise on four different surface momenta thereby giving the projected Weyl points an effective topological charge of \pm 2 . Hence, there must be two outgoing Fermi arcs for each Weyl-point projection. Moreover, we find that the projections of the TRI planes $k_{x}=k_{y}$ and $k_{x}=-k_{y}$ feature an odd number of surface Kramers pairs (see Fig. 3, and Fig. 2 in Ref. [44]). This implies nontrivial $\mathbb{Z}_{2}$ invariants which we have confirmed by explicit calculations using the Wannier-center formulation of the topological number (see Ref. [44]). This gives rise to restrictions on the Fermi surface topology. In particular, we find that we can tune between different Fermiarc connectivities by varying the Fermi level (see Fig. 3), which can be accomplished, for instance, by doping.

In Fig. 3(a), the Fermi level coincides with the Weylpoint energies. In this case, the Fermi arcs connect in a way that does not require an additional Fermi pocket. By raising the Fermi level, a Lifshitz transition takes place [compare Fig. 3(b) to Fig. 2(c)]. Finally, the connectivity of the Weyl nodes switches, which leads to the emergence of an additional Fermi pocket around the projected $\Gamma$ point, as shown in Fig. 3(c). This Fermi pocket is, indeed, associated with a surface Dirac cone [see Fig. 3(d)] as one can infer from surface Fermi surfaces at larger $E_{F}$ and from the dispersion along high-symmetry cuts through the surface BZ (see Ref. [44]).

Conclusions.-Besides the topological charges of the Weyl nodes, a generic TRI Weyl semimetal can be characterized by six $\mathbb{Z}_{2}$ invariants associated with the TRI planes of the 3D BZ. For the surface of a time-reversal invariant WSM, they impose restrictions on the number of surface Kramers pairs along the surface projections of the TRI planes and, therefore, also on the structure of the Fermi arcs which connect the Weyl nodes on the surface. Nevertheless, a remaining modulo-two ambiguity gives rise to many possible and qualitatively different Fermi-arc connectivities. In particular, certain connectivities require the creation of a Fermi pocket which is connected to the presence of a surface Dirac cone pinned to a TRI momentum. This changes the topology of the Fermi surface and is, thus, accompanied by a Lifshitz transition. It is crucial to note that this transition does not change the $\mathbb{Z}_{2}$ invariants and is, therefore, generic to all TRI Weyl semimetals. Further, we have shown that the coexistence of Fermi arcs and Dirac cones leads to universal, kidney-shaped features in QPI patterns which are accessible in scanning tunneling spectroscopy experiments. Finally, our density-functional theory calculations show that the half-Heusler compound LaPtBi under compressive strain is a good candidate material to realize the general coexistence established in this Letter. 
We acknowledge the financial support of the Future and Emerging Technologies (FET) Programme within the Seventh Framework Programme for Research of the European Commission under FET-Open Grant No. 618083 (CNTQC). This work has been supported by the Deutsche Forschungsgemeinschaft under Grants No. OR 404/1-1 and No. SFB 1143. C. O. acknowledges support from a VIDI grant (Project No. 680-47-543) financed by the Netherlands Organization for Scientific Research (NWO).

[1] K. v. Klitzing, G. Dorda, and M. Pepper, Phys. Rev. Lett. 45, 494 (1980).

[2] D. J. Thouless, M. Kohmoto, M. P. Nightingale, and M. den Nijs, Phys. Rev. Lett. 49, 405 (1982).

[3] M. Kohmoto, Ann. Phys. (N.Y.) 160, 343 (1985).

[4] X.-L. Qi and S.-C. Zhang, Rev. Mod. Phys. 83, 1057 (2011).

[5] M. Z. Hasan and C. L. Kane, Rev. Mod. Phys. 82, 3045 (2010).

[6] M. Sato and Y. Ando, Rep. Prog. Phys. 80, 076501 (2017).

[7] A. A. Burkov, Nat. Mater. 15, 1145 (2016).

[8] T. Senthil, Annu. Rev. Condens. Matter Phys. 6, 299 (2015).

[9] A. Lau, C. Ortix, and J. van den Brink, Phys. Rev. Lett. 115, 216805 (2015).

[10] C. L. Kane and E. J. Mele, Phys. Rev. Lett. 95, 226801 (2005).

[11] L. Fu, C. L. Kane, and E. J. Mele, Phys. Rev. Lett. 98, 106803 (2007).

[12] B. A. Bernevig, T. L. Hughes, and S.-C. Zhang, Science 314, 1757 (2006).

[13] M. König, S. Wiedmann, C. Brüne, A. Roth, H. Buhmann, L. W. Molenkamp, X.-L. Qi, and S.-C. Zhang, Science 318, 766 (2007).

[14] Y. Xia, D. Qian, D. Hsieh, L. Wray, A. Pal, H. Lin, A. Bansil, D. Grauer, Y.S. Hor, R. J. Cava, and M.Z. Hasan, Nat. Phys. 5, 398 (2009).

[15] C. Pauly, B. Rasche, K. Koepernik, M. Liebmann, M. Pratzer, M. Richter, J. Kellner, M. Eschbach, B. Kaufmann, L. Plucinski, C. M. Schneider, M. Ruck, J. van den Brink, and M. Morgenstern, Nat. Phys. 11, 338 (2015).

[16] Y. Ando and L. Fu, Annu. Rev. Condens. Matter Phys. 6, 361 (2015).

[17] L. Fu, Phys. Rev. Lett. 106, 106802 (2011).

[18] Y. Tanaka, Z. Ren, T. Sato, K. Nakayama, S. Souma, T. Takahashi, K. Segawa, and Y. Ando, Nat. Phys. 8, 800 (2012).

[19] A. Lau, J. van den Brink, and C. Ortix, Phys. Rev. B 94, 165164 (2016).

[20] S.-M. Huang, S.-Y. Xu, I. Belopolski, C.-C. Lee, G. Chang, B. Wang, N. Alidoust, G. Bian, M. Neupane, C. Zhang, S. Jia, A. Bansil, H. Lin, and M. Z. Hasan, Nat. Commun. 6, 7373 (2015).

[21] B. Q. Lv, H. M. Weng, B. B. Fu, X. P. Wang, H. Miao, J. Ma, P. Richard, X. C. Huang, L. X. Zhao, G. F. Chen, Z. Fang, X. Dai, T. Qian, and H. Ding, Phys. Rev. X 5, 031013 (2015).

[22] S.-Y. Xu et al., Science 349, 613 (2015).
[23] E. Haubold, K. Koepernik, D. Efremov, S. Khim, A. Fedorov, Y. Kushnirenko, J. van den Brink, S. Wurmehl, B. Buchner, T. K. Kim, M. Hoesch, K. Sumida, K. Taguchi, T. Yoshikawa, A. Kimura, T. Okuda, and S. V. Borisenko, Phys. Rev. B 95, 241108 (2017).

[24] X. Wan, A. M. Turner, A. Vishwanath, and S. Y. Savrasov, Phys. Rev. B 83, 205101 (2011).

[25] A. A. Burkov and L. Balents, Phys. Rev. Lett. 107, 127205 (2011).

[26] A. A. Zyuzin, S. Wu, and A. A. Burkov, Phys. Rev. B 85, 165110 (2012).

[27] T. Ojanen, Phys. Rev. B 87, 245112 (2013).

[28] A. A. Soluyanov, D. Gresch, Z. Wang, Q. Wu, M. Troyer, X. Dai, and B. A. Bernevig, Nature (London) 527, 495 (2015).

[29] R. Okugawa and S. Murakami, Phys. Rev. B 89, 235315 (2014).

[30] A. G. Grushin, J. W. F. Venderbos, and J. H. Bardarson, Phys. Rev. B 91, 121109 (2015).

[31] S. Juergens and B. Trauzettel, Phys. Rev. B 95, 085313 (2017).

[32] J. Ruan, S.-K. Jian, H. Yao, H. Zhang, S.-C. Zhang, and D. Xing, Nat. Commun. 7, 11136 (2016).

[33] P. G. Derry, A. K. Mitchell, and D. E. Logan, Phys. Rev. B 92, 035126 (2015).

[34] S. Kourtis, J. Li, Z. Wang, A. Yazdani, and B. A. Bernevig, Phys. Rev. B 93, 041109 (2016).

[35] G. Chang, S.-Y. Xu, H. Zheng, C.-C. Lee, S.-M. Huang, I. Belopolski, D. S. Sanchez, G. Bian, N. Alidoust, T.-R. Chang, C.-H. Hsu, H.-T. Jeng, A. Bansil, H. Lin, and M.Z. Hasan, Phys. Rev. Lett. 116, 066601 (2016).

[36] R. Batabyal, N. Morali, N. Avraham, Y. Sun, M. Schmidt, C. Felser, A. Stern, B. Yan, and H. Beidenkopf, Sci. Adv. 2, e1600709 (2016).

[37] H. Zheng et al., ACS Nano 10, 1378 (2016).

[38] H. Nielsen and M. Ninomiya, Nucl. Phys. B193, 173 (1981).

[39] C.-K. Chiu, J. C. Y. Teo, A. P. Schnyder, and S. Ryu, Rev. Mod. Phys. 88, 035005 (2016).

[40] G. Chang, D. S. Sanchez, B. J. Wieder, S.-Y. Xu, F. Schindler, I. Belopolski, S.-M. Huang, B. Singh, D. Wu, T. Neupert, T.-R. Chang, H. Lin, and M. Zahid Hasan, arXiv:1611.07925.

[41] L. Fu and C. L. Kane, Phys. Rev. B 74, 195312 (2006).

[42] J. E. Moore and L. Balents, Phys. Rev. B 75, 121306 (2007).

[43] R. Roy, Phys. Rev. B 79, 195322 (2009).

[44] See Supplemental Material at http://link.aps.org/ supplemental/10.1103/PhysRevLett.119.076801 for a detailed density-functional theory study of LaPtBi. Furthermore, we present the analytical treatment of the JDOS, the Wannier-center method used for the calculation of $\mathbb{Z}_{2}$ invariants, the iterative scheme used for the calculation of surface Green's functions, a discussion on the surface spectra of the tight-binding model, and a generalization to Weyl semimetals with more than four Weyl nodes. We also include a more detailed argument regarding the connection between Fermi pockets and surface Dirac cones and elaborate on why the six $\mathbb{Z}_{2}$ invariants are independent for time-reversal invariant WSMs.

[45] G. E. Volovik, Low Temp. Phys. 43, 47 (2017). 
[46] A. Varlet, M. Mucha-Kruczyński, D. Bischoff, P. Simonet, T. Taniguchi, K. Watanabe, V. Fal'ko, T. Ihn, and K. Ensslin, Synth. Met. 210, 19 (2015).

[47] L. Simon, F. Vonau, and D. Aubel, J. Phys. Condens. Matter 19, 355009 (2007).

[48] A. K. Mitchell and L. Fritz, Phys. Rev. B 93, 035137 (2016).

[49] H.-M. Guo and M. Franz, Phys. Rev. B 81, 041102 (2010).
[50] R. Yu, X. L. Qi, A. Bernevig, Z. Fang, and X. Dai, Phys. Rev. B 84, 075119 (2011).

[51] M. P. L. Sancho, J. M. L. Sancho, and J. Rubio, J. Phys. F 15, 851 (1985).

[52] D. Xiao, Y. Yao, W. Feng, J. Wen, W. Zhu, X.-Q. Chen, G. M. Stocks, and Z. Zhang, Phys. Rev. Lett. 105, 096404 (2010).

[53] K. Koepernik and H. Eschrig, Phys. Rev. B 59, 1743 (1999). 